\title{
Cathode Modification Method for Electrolytic Machining of Face Gear
}

\section{Yongming Wang ${ }^{1, a}$, Yan Cao ${ }^{2 *}$, b, Yaru Shi ${ }^{3}$, cand Liang Huang ${ }^{4, d}$}

1, 2, 3,4 Department of Mechanical and Electronic Engineering, Xi'an Technological University, Xi'an, China

axiangxiang2050@163.com, ${ }^{b}$ jantonyz@163.com, ${ }^{c}$ shi901006@163.com, ${ }^{d i m b a 11 @ 163 . c o m ~}$

\section{Keywords: Electrolytic machining; Cathode modification; Modification coefficients}

\begin{abstract}
In view of the difficulty of cathode modification, this paper proposes a new method based on the theory of equilibrium gap and cathode modification. Firstly, the mathematical model of cathode modification of face gear is established and different normal vectors of cathode surface are selected. Then in the process of electrolytic machining, the selecting method of modification coefficients is put forward. Compared with conventional technology, the proposed method can reduce the repair cycle and cost.
\end{abstract}

\section{Introduction}

Face gear is a kind of plane gear meshing with cylindrical gear. Due to its capacities of high speed and heavy load transmission, it is widely used in the field of aerospace and other high-speed precise transmission [1, 2]. The tooth flank of face gear is a kind of complex surface. Furthermore traditional face gear processing method is not only high cost but also time consuming. However as a non-contact special processing technology, electrolytic machining has the advantages such as high efficiency, better quality and the lossless of cathode tool. Therefore it can solve the problems existed in the process of traditional method [3,4]. Conventional cathode modification method is not able to deal with the mapping problem (i.e. modification coefficient) of theory gap and actual gap. Thus in this paper, a mathematical model of cathode modification is established and different normal vectors of cathode surface are selected. Meanwhile, the method of calibrating multiple modification coefficients is also proposed.

\section{Modification Principle}

With the improvement of the accuracy requirement of face gear, the processed workpiece of initial cathode is always not able to meet the requirements of actual engineering application. It needs to be modified with the cathode. The purpose of cathode modification is to check the tooth surface errors of the processed anode (specimen). If the accuracy is not satisfied, it is fed into the modification model and the correction of cathode surface (compensation amount of machining gap) is calculated. Then the cathode model is regenerated, and electrochemical machining process is implemented until the precision of the product meets the requirement [5]. The repair process of cathode is shown in Fig. 1.

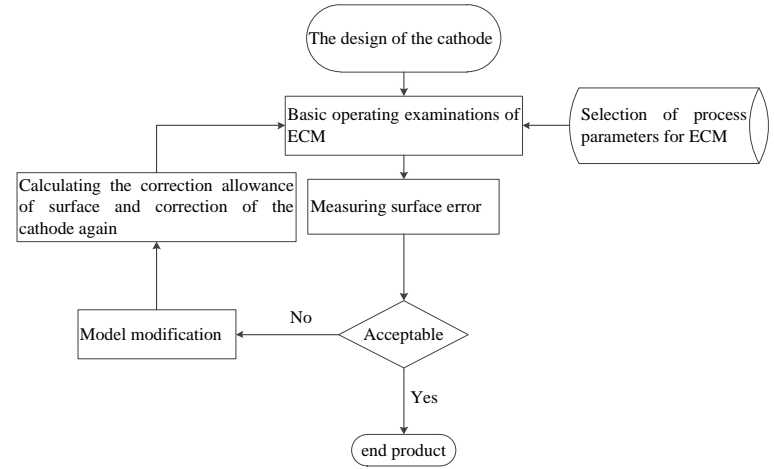

Figure 1. modification process of cathode 
In the process of cathode modification, Eq. 1 and the corresponding compensation amount of sampling points in the surface of cathode are utilized to obtain the cathode modification coordinate value ( $\left.\mathrm{xc}^{\prime}, \mathrm{yc}^{\prime}, \mathrm{zc}^{\prime}\right)$. The calculation formula is as Eq. 2. Three coordinate measuring instruments is used to measure the corresponding coordinate point of the tooth surface of workpiece. Then the compensation amounts of the corresponding clearance of sampling points are obtained. Eq. 2 is applied to reconstruct the corresponding three-dimensional model, and another cathode modification is completed. In the meantime, experiment is carried out.

$$
\begin{aligned}
& \left\{\begin{array}{l}
x_{c}=x_{a}-\Delta_{s} \cos \alpha \\
y_{c}=y_{a}-\Delta_{s} \cos \beta \\
z_{c}=z_{a}-\Delta_{s} \cos \gamma
\end{array}\right. \\
& \left\{\begin{array}{l}
x_{c}{ }^{\prime}=x_{a}-\left(\Delta_{s}+d\right) \cos \alpha \\
y_{c}{ }^{\prime}=y_{a}-\left(\Delta_{s}+d\right) \cos \beta \\
z_{c}{ }^{\prime}=z_{a}-\left(\Delta_{s}+d\right) \cos \gamma
\end{array}\right.
\end{aligned}
$$

Where $\mathrm{xc}, \mathrm{yc}, \mathrm{zc}$ denotes the coordinate value of cathode tooth surface, xa, ya, za is the coordinate value of the tooth surface sampling points of the anode workpiece, $\alpha, \beta, \gamma$ is the angle between the coordinate axes $\mathrm{x}, \mathrm{y}, \mathrm{z}$ and the sampling points of the straight tooth surface gear.

Where $\mathrm{xc}^{\prime}, \mathrm{yc}^{\prime}, \mathrm{zc}^{\prime}$ denotes the coordinate values of the sampling points for another modification, $\mathrm{d}$ is the correction of corresponding sampling points of cathode tooth surface.

\section{Establishment of Cathode Modification Model}

In the process of cathode modification of face gear, it is critical to establish appropriate mathematical model of cathode. It means that the relationship between the processing error and the correction errors is determined. Four section lines of the mathematical model are respectively selected including the initial designed cathode model, the corresponding cathode model after cathode modification, the corresponding theoretical model (face gear model) and workpiece after electrolytic process. The established mathematical model of cathode modification is shown in Fig. 2 .

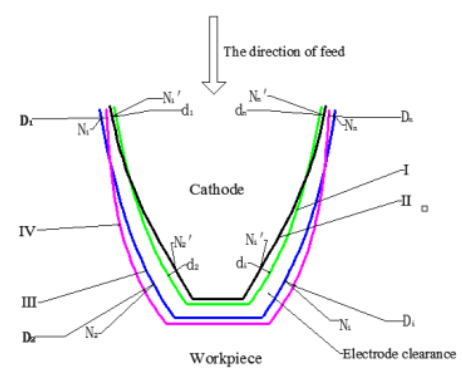

Figure 2. Schematic diagram of cathode modification

In Fig. 8, I is the section line of the initial designed cathode model. II is the section line of the cathode model after another cathode modification. III is the section line of the theoretical model (straight tooth surface gear model). IV is the corresponding section line of the work piece after the process of electrolysis. D1, D2, Di...Dn is the error value between the tooth surface section and the corresponding section of the theoretical model. $\mathrm{d} 1 \mathrm{~d} 2 \mathrm{di}$... dn represents the clearance compensation value of cathode modification. 
Due to the error between III and IV, cathode modification needs to be performed. Taking the point of the theoretical model as an example, the clearance between theoretical and actual is Di. The compensation clearance of corresponding points in the cathode is di. Then a new cathode sample point NY's achieved. In the same way, a series of sample points are obtained and the section line of the cathode model after modification is formed. The relationship between the error values of points on section line of the workpiece and corresponding cathode profile is described as Eq. 3.

$d_{i}=\varepsilon D_{i} i=1,2, \ldots n$

Where $\varepsilon$ is modification coefficient and the experience value is $0.1 \sim 1$ (according to the specific process parameters).

There are two critical points in the process of cathode modification. Specifically, it includes the determination of modification coefficient by finding the quantitative relationship between parts processing error and the corresponding clearance compensation amount, and the determination of coordinates of points on the corrected cathode surface [5].

\section{Treatment of Cathode Modification Ratio Coefficient $\varepsilon$}

When cathode is modified, the operator detects the machining error through special measuring tool. Thus the corresponding parts of cathode surface are repaired by hand [6]. In the process of artificial modification, the selection of modification coefficient has great dependence on the experience of operator's electrochemical machining. Furthermore the cathode has a large randomness, which affects the precision of the machining parts.

In order to avoid the above situation, the numerical control machine is used for digital correction and the correction process is realized by computer. The precision of cathode repeat modification is improved effectively [7]. Three coordinate measuring machines are used to measure the sampling points before machining. And the data points of surface are obtained which corresponding with the sampling points in the theoretical model. According to the practical experience, the repair process includes the determination of modification coefficient, processing, measurement, feedback; adjust the modification coefficient and another processing. Until the work piece is processed to meet the requirements, and improve the precision of the machining parts [8].

In the process of measurement, normal direction of the tooth surface is measured and the choice of normal vector is directly influence the $\mathrm{Di}$ and di. Here are two means to select the normal vector.

(1) According to the normal vector of sampling points on the tooth surface (3D model), and it is taken as the correct direction. However, corresponding modification is based on the normal vector direction of the initial cathode model. Each modification is based on the theoretical model and the initial model [9].

(2) The 3D model of tooth surface is reconstructed based on the data of measured points after the first modification of tooth surface. And the normal vector of the tooth surface is used as the correct direction. The choice of the normal vector of the corresponding modification is also based on the the method. In this way, the last time data is used to iteration for each modification.

Compared the two kinds of means, the second is able to effectively use the data on the basis of last iteration and achieve qualified cathode faster. However, it also has drawbacks such as it is necessary to rebuild a three-dimensional model after the measurement. Because isolated point has not normal vector. Therefore the requirement of modeling is relatively enhanced.

Due to the complexity of electrochemical machining, it is difficult to find a definite analytic expression to describe the regularity between the above two. Electrolytic processing needs to be verified by many experiments. And how to shorten the test cycle is a key problem. In order to settle this problem, this paper proposes a method by simultaneously validating multiple scale coefficients [10].

(1)Meshing division including 63 points and 40 surfaces is implemented while the 3D model is established. As it is shown in Fig. 3, the tooth surface is divided into four regions and four ratio 
coefficients $\square 1, \square 2, \square 3, \square 4$ are selected. And the corresponding correction coefficients of each region are $0.25,0.5,0.75$, and 1 .

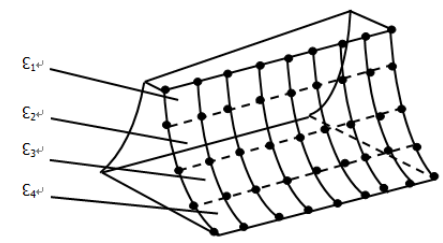

Figure 3. Partition coefficient

(2)The experiment is carried out, and three coordinates instrument is used to measure the processed parts. Corresponding sampling points are measured. Modeling is performed through UG, and the errors are compared. In the meantime, the optimal modification coefficient (the smallest error) is found out and the four ratio coefficients can be verified one time.

(3)Then the best modification coefficient $\square 2$ is divided into four regions, such as $\square 21, \square 22$, $\square 23, \square 24$ Continue the electrochemical machining test, and the optimal proportion parameters are selected.

(4)While the ratio coefficient error is less than 0.05 , the optimal modification coefficient is selected and several correction tests are performed to verify the reasonableness of ratio coefficient. It is shown in Fig. 4.

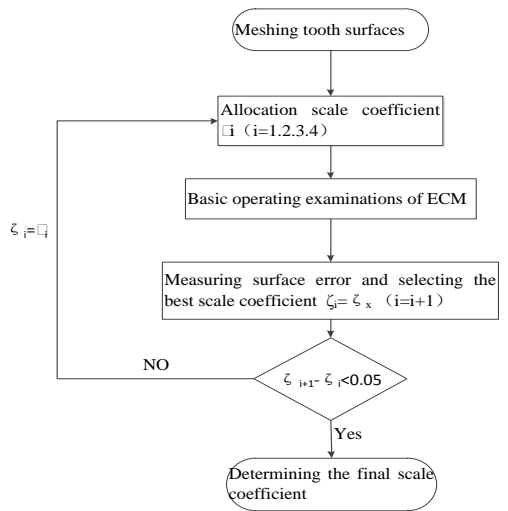

Figure 4. Selection of proportion coefficient

\section{Conclusion}

In this paper, the mathematical model of the cathode modification of face gear is established. Different normal vectors of tooth surface are selected to realize the effective iterative modification by using of the modification data. Through combining the division of tooth surface area, the method is proposed by simultaneously validating multiple proportion coefficients. It shows that the cathode modification cycle and the cost of modification can be reduced. Thus it provides an important guidance for the actual production.

\section{Acknowledgements}

This project is supported by the special processing Key Laboratory of Shaanxi province (15JS041). The project name is "the key technology research of multi axis NC machining of spur gear.

\section{References}

[1] Litivin F L, Zhang Y, Wang J C, et al. Design \& Geometry of Face Gear Drive[J]. Journal of Mechanical Design, 1992, 114(4): 642-647. 
[2] Litivin F L. Handbook of face gear drives with a spur Involute pinion [A]. NASA Fianal Contractor Report CR-209909[C]. Canada: [sn], 2000. 345-353.

[3] Zhu Di. The foreign research progress of electrochemical machining [J]. ELECTROMACHINING \& MOULD, 2000, 5(1):10-12.

[4] Wang Lei. Tool Design of Electrochemical Machining with Passivating Electrolyte in Aero engine B lades [J]. Mechanical Science and Technology, 2006, 25(2):229-230.

[5] Zhu Dong. Experimental Study on the Cathode Digital Modification of Turbine Blade in Electrochemical Machining [J]. Journal of Mechanical Engineering, 2011, 47(7):192-193.

[6] Wang Lei. The cathode design of Turbine Blade in Electrochemical Machining [C]// Proceedings of the 2005 Annual Conference of China Mechanical Engineering Society. Beijing: Machinery Industry Press, 2005:250-252.

[7] Su Jianxin. Optimal Tooth Surface Correction of Internal Helical Gear and Mesh Simulation [J]. Journal of Mechanical Transmission, 2015, 39(7):7-10.

[8] Tang Xinglun. Boundary Adjusting Method for Cathode Design in Electrochemical Machining [J]. China Mechanical Engineering, 2004, 15(2):106-109.

[9] Yang Xinru. Gear Modification Design Considering Axis Parallelism Deviation and Thermal Deformation [J]. Journal of Mechanical Transmission2016, 40(10):75-77.

[10] Jin Tingting. Analysis on Contact and Relative Fatigue Life of Logarithmic Crowned Helical Gears with High Contact Ratio [J] Mechanical Science and Technology for Aerospace Engineering, 016, 35(6) 962-966. 